\title{
Sobre o DeSEnVolvimento Histórico do ConCEITo de NúMERo
}

\author{
Inocêncio Fernandes Balieiro Filho \\ Universidade Estadual Paulista “Júlio de Mesquita Filho” - UNESP - Brasil
}

(aceito para publicação em maio de 2016)

\begin{abstract}
Resumo
Este artigo apresenta um panorama histórico do desenvolvimento do conceito de número, buscando contribuir para o conhecimento e a cultura do professor de Matemática e para o seu trabalho em sala de aula. Para isso, por meio da historiografia, foram pesquisados e selecionados textos de matemáticos e filósofos de diferentes períodos da história que tiveram a preocupação em definir o número e consultadas obras de historiadores da Matemática.
\end{abstract}

Palavras-chave: Número, História, Filosofia da Matemática.

\section{[About History of Number Concept Development]}

\begin{abstract}
In this paper we present a historical view about number concept development to contribute to teacher knowledge and the culture and to their work in classroom. For that, using historiography methodology in research, we select mathematics and philosophy works in different history period that have a concern to define number and we consult mathematics history works.
\end{abstract}

Keywords: Number; History; Philosophy of Mathematics.

\section{Introdução}


Os números são criações livres do intelecto humano; eles servem como um meio de apreender mais facilmente e de forma mais acentuada a diversidade das coisas. Julius Wilhelm Richard Dedekind (1831 - 1916)

Nos diferentes níveis de ensino, ao se ensinar Matemática, o número é um assunto presente, ainda que sejam diferentes as formas de apresentação e os níveis de abstração. Nas séries iniciais, a noção de número está relacionada com processos de contagem e medidas. Nas séries intermediárias e no Ensino Médio, o número assume uma característica algébrica e é explorada a sua construção valendo-se de relações de equivalência e de ordem. Já em níveis avançados, valendo-se dos axiomas de G. Peano (1858-1932), é caracterizado o conjunto dos números naturais e, com base nesse conjunto, são construídos os conjuntos dos números inteiros e dos números racionais. E, finalmente, com a definição de corpo ordenado que satisfaça o axioma da completude, é construído o conjunto dos números reais.

No transcorrer da História da Matemática, diferentes matemáticos e filósofos preocuparam-se em estabelecer uma definição para "número", por ser esse um elemento básico no desenvolvimento de uma teoria. A definição de número é uma das questões dos Fundamentos da Matemática que ocuparam e ainda ocupam os filósofos da Matemática.

Thompson (1992), em suas investigações sobre as concepções que os professores têm sobre a Matemática, aponta que esses têm uma cultura Matemática reduzida, isto é, sabem pouco sobre a História e a Filosofia dessa ciência, e isso faz que tendam para uma visão absolutista e instrumental da Matemática, considerando-a como uma acumulação de fatos, regras, procedimentos e teoremas. Fennema e Leof (1992) apresentam vários exemplos que sugerem que o conhecimento e a cultura matemática do professor podem ter influência no seu estilo de ensino.

Neste trabalho, apresentamos um panorama histórico do desenvolvimento da definição de número, buscando contribuir para o conhecimento e a cultura do professor de Matemática. Conforme Felix Klein, "esperamos que discussões deste tipo contribuam para a cultura matemática geral: ao lado do conhecimento de detalhes específicos (técnicas), que se adquire nas várias disciplinas, deve haver uma compreensão dos conteúdos e das relações históricas existentes entre eles." (Klein, 2004, p.2).

A história e a filosofia devem ter lugar de destaque nas disciplinas do curso de Matemática, já que ambas contribuem para um entendimento mais amplo dos conceitos que fazem parte dessa ciência e essa compreensão contribuirá para o trabalho do professor em sala de aula.

\section{Sobre a Metodologia da Pesquisa}

Para a elaboração do presente estudo tomou-se como base as etapas de pesquisa em História sugeridas por Besselaar (1973) e os critérios de classificação de fontes históricas indicados por May (1978).

Dadas essas referências, buscou-se adaptá-las às características da pesquisa proposta para esse trabalho. Foram seguidas sete etapas: 1. Levantamento Bibliográfico, com o intuito de encontrar os livros, artigos e documentos que estivessem relacionados com 
o assunto da Pesquisa; 2. Documentação, para a distinção entre as fontes primárias (obras originais) e as fontes secundárias (obras dos historiadores); 3. Leitura de Reconhecimento, para a coleta de dados referente ao tema pesquisado, com o objetivo de verificar a existência ou não das informações que estão sendo procuradas; 4. Leitura Seletiva, para a localização das respostas, em cada texto, para as questões do tema da pesquisa; 5. Leitura Reflexiva, com o propósito de compreender o que o autor assevera sobre o assunto; 6 . Leitura Interpretativa, partindo dos objetivos do pesquisador e do tema pesquisado, buscase esclarecer o que o autor declara em relação ao tema pesquisado. Nessa etapa se estabelece a relação entre o que o autor expõe no texto e o objetivo do pesquisador e são determinadas as verdades que se apresentam de forma perceptível e patente que compelem o pesquisador a uma segura anuência a respeito do assunto pesquisado; 7. Finalmente, procede-se a Redação da Pesquisa. (Maiores detalhamentos das etapas de pesquisa podem ser vistas em Balieiro, 2004, p. 10 a 17.).

\section{O Desenvolvimento Histórico do Conceito de Número}

Ainda que os egípcios e os babilônios tenham desenvolvido um sistema numérico, não há registros históricos que comprovem um interesse dessas civilizações em estudar o significado filosófico do número. Mas vale destacar que os egípcios usavam um sistema de numeração, ambos baseados em agrupamento de dez. Suas operações aritméticas básicas eram a adição e a duplicação e para multiplicar ou dividir desenvolveram um método que utilizava a duplicação.

No caso dos números racionais, os egípcios trabalharam com a ideia de "uma nésima parte", ou seja, as frações unitárias. Como a duplicação era importante em sua Matemática, o trabalho com as frações unitárias não era fácil. Dessa forma, eles elaboraram listas de dobros das partes. Por exemplo, o dobro de um quinto (que é dois quintos), era expresso como a soma entre um terço e um quinze avos.

Já os babilônicos representavam os números usando um sistema numérico posicional de base 60 e estenderam esse sistema para tratar das frações. Do mesmo modo que eles escreviam 72 (com seus símbolos) como "1,12" - significando 1 x $60+12$, escreviam $72 \frac{1}{2}$ como " 1,$12 ; 30$ " - significando 1 x $60+12+30$ x 1/60.

Ainda assim, como enfatiza Struik, "As matemáticas orientais surgiram como uma ciência prática com os objetivos de facilitar o cálculo do calendário, a administração das colheitas, a organização das obras públicas e a cobrança de impostos.” (Struik, 1992, p.47). Além disso, "as matemáticas mesopotâmicas atingiram um nível mais elevado do que o obtido pelas matemáticas egípcias" (Struik, 1992, p.56).

Além disso, Reale e Antiseri afirmam:

"Está demonstrado historicamente que os povos orientais com os quais os gregos tinham contato possuíam verdadeiramente uma forma de 'sabedoria', feita de conviç̧ões religiosas, mitos teológicos e 'cosmogônicos', mas não uma ciência filosófica baseada na razão pura (no logos, como dizem os gregos). Ou seja, possuíam um tipo de 
sabedoria análogo a que os próprios gregos possuíam antes de criar a filosofia”. (REALE e ANTISERI, 1990, p.12-13).

Ainda que citemos Struik e Reale \& Antiseri, devemos deixar explícito o nosso respeito pelos empreendimentos realizados pelos egípcios e babilônios na matemática,

"pois esses mostram um considerável avanço em processos aritméticos, algébricos e geométricos. De fato, ao avaliar os problemas descritos nos papiros egípcios e nas plaquetas de argila dos babilônicos, observa-se que essas civilizações utilizaram a aritmética, a álgebra e a geometria para diversos propósitos: no comércio e na administração, na medição de superfícies de terrenos, para estimar a produção agrícola, no cálculo de volumes de reservatórios e da quantidade de ladrilhos ou blocos de rochas necessários para construir um templo ou uma pirâmide e no estudo da astronomia com o intuito de confeccionar calendários astronômicos para satisfazer suas necessidades domésticas, comerciais e religiosas". (BALIEIRO e REICHER, 2008, p.157)

\section{A visão de Número dos Gregos e Árabes}

Os primeiros filósofos gregos desenvolveram um pensamento reflexivo sobre o como e o porquê das coisas, não estando interessados em aplicações práticas. Conforme registros históricos, o primeiro homem, precursor desse pensamento crítico e fundador da escola jônica, foi Tales de Mileto (624-547 a.C.), que junto com Anaximandro de Mileto (611-546 a.C.) e Anaxímenes de Mileto (585-525 a.C.) iniciou um pensamento filosófico que incidia sobre a natureza, ou seja, começou a realizar reflexões importantes na orientação de atividades relacionadas ao que hoje denominamos ciência natural. Conforme Heath (2003), a primeira definição de número é atribuída a Tales de Mileto, que o definiu como uma coleção de unidades ( $\mu$ ovó $\delta \omega v \sigma v i \sigma \tau \eta \mu \alpha)$.

O próximo a iniciar uma nova escola filosófica foi Pitágoras de Samos (569-475 a.C.), que é considerado um personagem importante do início do desenvolvimento da filosofia e da matemática grega. Os pitagóricos consideravam importante o que se ensinava do homem, da natureza da alma humana e suas analogias com outras formas de vida e as relações que se estabelecem com essa tríade. Para eles, o conceito de número tinha um significado místico e independente da realidade, isto é, o número era responsável pelo princípio harmônico que rege a estrutura do cosmo.

Os pitagóricos concebiam o número tomando-se por base a unidade, e alguns deles o denominavam como "uma progressão de multiplicidade iniciando pela unidade e uma regressão terminando nela". (Heath, 2003, p.70).

Para Platão (429-347 a.C.) o estudo da matemática tinha como objetivo o treinamento da mente e o seu valor prático não tinha importância em sua concepção filosófica. Katz (1998) afirma que, nesse período, os geômetras e filósofos fizeram pesquisas significativas em matemática na Academia de Platão, que reuniu estudiosos de 
várias partes do mundo grego. Dentre eles, destaca-se Eudoxo de Cnido (408-355 a.C.) que

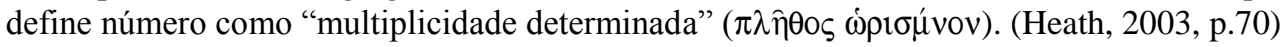
Outro discípulo de Platão, que se tornou filósofo notório, foi Aristóteles (384-322 a.C.), que começou a estudar na Academia com 18 anos e permaneceu lá até a morte de Platão. No que se refere à matemática sua influência maior foi na área da lógica. Para Aristóteles, em sua Metafísica: "a unidade não é um número; o termo "um" significa que é uma medida de alguma multiplicidade e o"número"que é uma multiplicidade de medidas." (Heath, 1970, p.83-84). E, Aristóteles (1862), em sua Física, no livro 9, define o número como uma pluralidade que se mede pela unidade.

Para Katz (1998), a obra matemática mais importante do período dos gregos e, provavelmente, de todos os tempos, denomina-se os Elementos de Euclides de Alexandria (325-265 a.C.). Esse trabalho fornece um modelo de como a"matemática pura" deveria ser estruturada, isto é, com base em axiomas, postulados, definições precisas, teoremas cuidadosamente indicados e demonstrações logicamente coerentes. Nesta obra, Euclides, no livro VII, definição 1 e 2, respectivamente, escreve: "Unidade é aquilo segundo o qual cada uma das coisas existentes é dita uma. E número é a quantidade composta de unidades." (Euclides, 2009, p. 269).

Katz (1998) afirma que quase nada se conhece sobre a vida do filósofo neopitagórico Nicômaco de Gerasa (60-120 d.C.). Sabe-se que provavelmente estudou filosofia e matemática em Alexandria e que seus tratados estão impregnados com as concepções de Pitágoras. Dois de seus trabalhos, Introdução à Aritmética e a Introdução à Harmônica sobreviveram. Na Introdução à Aritmética, Nicômaco, estabelece que: "Número é uma multiplicidade determinada ou coleção de unidades ou fluxo de quantidade composta de unidades." (Ivor, 1957, p.73)

Burton (2011) considera que, diante da descoberta dos números irracionais, a matemática grega afastou-se da abordagem puramente aritmética, o que fez com que todos os problemas algébricos fossem desenvolvidos utilizando um inadequado esquema geométrico. Diofanto de Alexandria (200-284), em sua Aritmética, rompe com esse enfoque e determina um marco histórico na evolução da álgebra ao introduzir um novo procedimento para resolver antigos e novos problemas algébricos. Em sua obra Aritmética, no livro I, lê-se: “(...) o número que não possui algumas das particularidades precedentes, mas que possui em si uma quantidade indeterminada de unidades chama-se "arithmós" e sua marca distinta é $\zeta^{1}$. " (Diophante, 1926, p.2).

$\mathrm{Na}$ matemática árabe encontramos definições dadas por dois matemáticos. AlKhawarizmi (780-850), escreve: "Eu também observei que cada número é composto de unidades e que qualquer número pode ser dividido em unidades." (Mohammed Ben Musa, 1831, p.5). Já para o matemático Omar Khayyam (1048-1122): "esta coisa conhecida é uma quantidade ou uma razão individualmente determinada" (Alkhayyami, 1851, p.5).

\section{A visão de Número a partir do Século XVI}

1 Letra grega; Sigma minúsculo que só é utilizado no final de uma palavra.

RBHM, Vol. 16, nº 31, p.51-63, 2016 
$\mathrm{Na}$ época considerada por alguns historiadores (Katz, 1998, Burton, 2011) como o começo da Matemática Moderna, S. Stevin (1548-1620), em sua obra L'Arithmetique, de 1585, apresenta uma definição de número. Ele escreve: "Definição I - A Aritmética é a ciência dos números. Definição II - Número é aquilo para o qual se explica a quantidade de cada coisa." (Stevin, 1585, p.1). Sua definição, de certa forma, segue o padrão da definição dada por Euclides. Pode-se perceber que há uma mudança em sua concepção de número em relação à estabelecida por Aristóteles, isto é, não há uma distinção entre número e magnitude.

No período de I. Newton (1643-1727) e G. W. Leibniz (1646-1716), a definição de número continua a ser foco de atenção entre os matemáticos, em face de alguns tratados publicados sobre Aritmética e Álgebra. De fato, em sua Universal Arithmetick, Newton define: "Por número entendemos tanto uma multiplicidade de unidades, como a razão abstraída de qualquer quantidade para outra quantidade de mesma espécie, que tomamos para a unidade." (Newton, 1720, p.2)

Pode-se salientar, conforme Bashmakova e Smirnova (2000), que na Europa do século XVI e XVII havia ainda uma preponderância da tradição euclidiana, porém, com essa definição, Newton foi o primeiro a romper com a tradição euclidiana, em especial, com a concepção de número (quantidade composta de unidades), pois a razão de números (números racionais) e a razão de quantidades (números reais) não eram consideradas como números na Antiguidade.

Já a concepção de número de Leibniz, tinha um caráter mais filosófico. De fato, em sua Oeuvres Philosophiques, pode-se ler:

Nos números as ideias são mais precisas e mais próprias de serem distinguidas umas das outras do que em seu significado, em que não se pode observar ou medir cada igualdade e cada excesso de grandeza tão facilmente como nos números, pela razão que no espaço não saberíamos chegar pelo pensamento a certa pequenez determinada além do que não poderíamos ir como é a unidade no número. (LEIBNIZ, 1765, p.113)

Num contexto filosófico amplo, a noção de número em Leibniz, segundo Brown e Fox (2006), pode ser entendida tomando-se por base dois números fundamentais: o 0 e o 2 , já que ele concebeu as verdades das aritméticas como verdades eternas, cujo princípio maior residia na contradição. Qualquer verdade aritmética poderia ser reduzida a uma declaração de identidades por meio de uma sequência de definições, ou seja, "2" pode ser definido como " $1+1$ ", " 3 " como " $1+2$ ", e assim por diante. Portanto, qualquer soma correta seria redutível a uma declaração de identidades.

De acordo com Burton (2011), o século XVIII foi denominado e descrito como a Era do Iluminismo. Esse termo caracteriza o curso das ideias liberais que buscavam melhorar as condições práticas da vida humana por meio do poder da razão. Embora essas concepções fossem amplamente disseminadas na Europa, seu principal foco intelectual estava na França, nas obras de alguns pensadores como Montesquieu (1689-1755), D. Diderot (1713-1784), J. R. d'Alembert (1717-1783), J. J. Rousseau (1712-1778) e Voltaire 
(1694-1778). Nesse ambiente de efervescência intelectual, fértil de ideias e concepções revolucionárias nos vários campos do conhecimento, encontra-se L. Euler (1707-1783), o mais importante matemático dessa época.

Euler, em Éléments d'Algebre, define primeiramente grandeza e depois relação entre grandeza: "Denomina-se grandeza ou quantidade tudo o que é susceptível de aumentar ou de diminuir." (Euler, 1774, p.1).

"As determinações ou as medidas de grandezas de todas as espécies, retornam a esta: Que se fixar em primeiro lugar à vontade certa grandeza de mesma espécie que aquela que se quer determinar, a fim de tomar por medida ou unidade; então, que se determine a relação que a grandeza prescreve com essa medida conhecida. Esta relação se exprime sempre por números, donde se resulta que um número não é outra coisa que a relação de uma grandeza para com outra estimada arbitrariamente pela unidade." (EULER, 1774, p. 3-4).

D’Alembert contribuiu para enriquecer certos ramos da matemática, colaborou com Diderot na publicação da Encyclopédie e aproveitou para apresentar suas principais concepções matemáticas em diferentes artigos que aparecem nessa esplêndida obra. D’Alembert, na Encyclopédie Méthodique, define: "Número: diz-se vulgarmente em Aritmética de uma coleção ou reunião de unidades ou de coisas de mesma espécie." (D'Alembert \& Diderot, 1758, p.464)

Ainda no período de Euler, encontra-se o filósofo I. Kant (1724-1804) que, para expor suas concepções filosóficas sobre a Matemática, refletiu sobre o significado filosófico do número. Em seu tratado, Críticada Razão Pura, Kant define número como "uma representação que resume a adição sucessiva de uma unidade (homogênea) para outra." (Kant, 1905, p.136). Assim, parece que o número pressupõe o tempo e o espaço como o resultado de um relacionamento que implica não só a distinção dos objetos no espaço, mas também a sua sucessão no tempo.

Na transição entre os séculos XVIII e XIX, um dos matemáticos que se destaca é A. L. Cauchy (1789-1857), por introduzir inovações em diversas áreas da Matemática, ser o primeiro a estudar a teoria das funções analíticas, avançar de modo significativo nos estudos sobre a teoria de determinantes e ser o precursor em estabelecer o rigor na análise matemática. Na obra Cours d'Analyse, Cauchy define o que entende por número e quantidade:

"Para evitar toda espécie de confusão na linguagem e na escrita algébrica, vamos fixar nestes preliminares o significado de vários termos $e$ de várias notações que emprestaremos da álgebra ordinária ou da trigonometria. (...) Vamos indicar primeiro que ideia nos parece conveniente para ligar estas duas palavras: número e quantidade. Tomaremos sempre a denominação de número no sentido que se emprega em aritmética, ao fazer nascerem os números da medida absoluta das 
grandezas; e empregaremos unicamente a denominação de quantidades às reais positivas ou negativas, isto é, aos números precedidos dos símbolos + ou -." (CAUCHY, 1821, p.1-2)

O século XIX é marcado, especialmente, pelo surgimento da álgebra moderna. Ao longo desse século, os conceitos fundamentais da álgebra abstrata são consolidados, apresentando objetos de natureza distinta dos números reais ou complexos, ou seja, o estudo de algumas estruturas matemáticas com operações bem definidas. Segundo Katz (1998), os matemáticos ingleses do primeiro terço de século XIX, incluindo G.Peacock (1791-1858) e A. De Morgan (1806-1871), tentaram axiomatizar as ideias básicas da álgebra e determinar exatamente o quanto é possível generalizar as propriedades dos números inteiros para outros tipos de quantidades. Esse contexto levou W. R. Hamilton (1805-1865) à descoberta dos quatérnios, em 1843.

Com essas concepções inovadoras de novas estruturas algébricas, Peacock, em seu A Treatise on Algebra, define número:

"Os símbolos da Álgebra podem ser tomados como representantes de todas as espécies de quantidade, seja abstrata ou concreta: as operações a que estão sujeitos são perfeitamente gerais, e em nenhum aspecto são afetadas pela natureza das quantidades que os símbolos denotam, sendo determinadas exclusivamente pelas definições e pressupostos que constituem os primeiros princípios da ciência.” (PEACOCK, 1830, p.1.)

E, De Morgan, em Elements of Algebra, escreve:

"Em aritmética, usamos símbolos para números. Um símbolo é qualquer sinal de uma quantidade que não é a quantidade em si. Quando 1, 2, 3, etc., significa 1 milha, 2 milhas, 3 milhas, etc., ou 1 litro, 2 litros, 3 litros, etc., esses são chamados números concretos. Mas quando parte de toda ideia de 1, 2, etc., significando um, dois, etc., de qualquer coisa em particular como quando dizemos "seis e quatro faz dez", então os números são chamados números abstratos." (DE MORGAN, 1837, p.ii)

No final do século XIX e no início do século XX, nas discussões em Filosofia da Matemática, prevalecia a concepção de que a Matemática é uma ciência verdadeira. De um lado, os idealistas consideravam que toda a Matemática trabalhava simplesmente com aparências, ao passo que de outro, os empíricos sustentavam que toda a Matemática era uma aproximação a certa verdade exata sobre a qual nada tinham a dizer. Neste cenário conflitante, o estado conjetural e tumultuoso de concepções era completamente ingrato.

Mas, no começo do século XX, alguns matemáticos e filósofos da Matemática puderam contestar essas concepções, pelo menos até o ponto de reduzir todas as suas proposições a certas noções fundamentais de lógica. Sem se ocupar de derrubar ou de mover os elementos essenciais da lógica aristotélica, as investigações de G. Boole (1815- 
1864), C. S. Peirce (1839-1914), E. Schröder (1841-1902), G. Frege (1848-1925), A. N. Whitehead (1861-1947), B. Russell (1872-1970) e vários outros lógicos contribuíram com resultados para a elaboração do cálculo das classes e do cálculo proposicional, nos quais a teoria do silogismo aristotélico parece ocupar somente um espaço.

Nessa nova formulação, as possibilidades apresentadas pela lógica moderna, como instrumento científico, foram evidenciadas nas aplicações e investigações que se fizeram ao utilizá-la nos fundamentos da matemática.

Neste ponto a discussão foi retomada pela Filosofia da Matemática, que nesse contexto, procurou indicar quais são as noções fundamentais intrínsecas da Matemática e assinalar as dificuldades filosóficas envolvidas na análise da noção e definição de número.

As principais definições de número que foram desenvolvidas nesse período são a de J. W. R. Dedekind (1831-1916), J. F. L. P. Cantor (1845-1918) e Frege. Dedekind, em Essay on the Theory of Numbers, define:

"Se considerando um sistema $N$ simplesmente infinito, ordenados por uma aplicação $\varphi$, faz-se totalmente abstração da constituição particular dos elementos, que apenas retém o que os diferencia e que apenas se prende às relações que estabelece entre as aplicações $\varphi$ que define a ordem, vamos nomear estes elementos números naturais ou números ordinais ou simplesmente números, e o elemento fundamental 1 é chamado o número fundamental da sequência de $N$ números. Dada esta liberação de elementos de outros conteúdos (abstração), estamos autorizados a dizer que os números são uma livre criação do espírito humano." (DEDEKIND, 1901, p. 33).

Cantor (1883) define os números naturais como um caso particular dos números transfinitos. Em seu artigo Une Contribution a la Théorie des Ensembles, propôs uma definição geral de número inteiro, sejam finitos ou infinitos, na esperança de ver a comunidade matemática aceitar facilmente os números transfinitos. Desde o surgimento da noção de potência, ele insiste sobre sua característica global. Ele escreve: "Quando os conjuntos considerados são finitos (...), a noção de potência responde então àquela de número na significação de enumeração e, por conseguinte, também àquela de número inteiro positivo." (Cantor, 1883a, p.311). E acrescenta, em seu artigo Sur les Ensembles Infinis et Linéaires de Points: "A noção potência (...) abrange, como caso particular, a noção de número inteiro." (Cantor, 1883b, p. 363).

F. L. G. Frege, emThe Foundations of Arithmetic, define:

"O número não é abstração de objetos (...); não é uma propriedade dos objetos (...). A questão então permanece: quando se dá um número, o que sustenta nosso enunciado? O número não é um ser físico; mas ele não é subjetivo, ele não é uma representação. $O$ número não é gerado de adições de um objeto com outro, e a atribuição de um nome novo após cada uma dessas junções não faz uma convenção. As expressões "multiplicidade", "conjunto", "pluralidade" são, por suas 
indeterminações, inaptas para trazer algum esclarecimento sobre o número." (FREGE em BELNA, 1996, p. 227)

Finalizando essa revisão histórica, apresentamos as definições de B. Russell e J. von Neumann (1903-1957), pela contribuição às nossas discussões contemporâneas sobre o conceito de número.

Russell define: "Um número será um conjunto de classes tais que quaisquer duas são similares entre si e nenhuma fora do conjunto é similar a qualquer uma de dentro do conjunto.” (Russell, 1919, p.18-19). As ideias de lógica matemática de Peano e as preocupações de Frege em explicitar as noções entre os conceitos elementares e as proposições em Matemática influenciaram os trabalhos de Russel na elaboração da definição de número. A ênfase dada por Russell na formulação precisa dos conceitos fundamentais da Matemática e das suas relações com a lógica comprova tal influência.

Entretanto, o trabalho de Russell, que predomina na Filosofia da Matemática por quase um século, apresenta uma ideia ímpar: definir número mediante a noção de classes ou coleções.

Segundo Ewald (1999), com base nos sete axiomas propostos por E. Zermelo (1871-1953) para a formalização da Teoria de Conjuntos, von Neumann constrói sua própria teoria e, como consequência, elabora uma definição de número. Considerando $A$ como um certo campo de objetos abstratos, o axioma do infinito garante a existência em $A$ de um conjunto $B$ com os elementos $0,\{0\},\{\{0\}\}, \ldots$, e, von Neumann utiliza o conjunto enumerável de referência no sistema de Zermelo para representar a sequência $0,1,2, \ldots$ dos números naturais.

\section{Comentários}

Observamos que cada uma das definições aqui apresentadas é caracterizada pelo desenvolvimento matemático e filosófico presente em cada época.

Ao considerar o desenvolvimento histórico do conceito de número, podemos destacar diversos aspectos. Entre eles, aqui destaco quatro. O primeiro é a ideia de número como uma determinada quantidade de unidades, presente nas concepções dos matemáticos gregos. Essa ideia permanece com poucas modificações até àquela proposta por Newton, que considera como um número a razão de números (números racionais) e a razão de quantidades (números reais) e à proposta por Leibniz, que considera o número independente do que ele representa no mundo real, apresentando uma concepção de caráter filosófico. O segundo, é que o desenvolvimento da Álgebra no século XIX, sem dúvida, promoveu um avanço nas definições de número. O terceiro são as ideias de ordem e aplicação, presente na definição proposta por Dedekind e influenciada pelas ideias de Peano. E, o quarto, é a influência das ideias de conjunto elaboradas por Cantor na definição proposta por Russell e, posteriormente, na proposta por von Neumann.

Outro ponto importante que devemos ressaltar, é que os números são parte do nosso cotidiano e, por isso, muitas vezes nos esquecemos dos esforços de vários matemáticos e filósofos que, durante séculos, estiveram envolvidos com o seu desenvolvimento e com as questões sobre os fundamentos da Matemática. 
Percebemos que este desenvolvimento foi iniciado em tempos remotos, quando os números naturais surgiam naturalmente a partir da necessidade do homem de contar e, posteriormente, das relações comerciais. Porém, ainda que os egípcios e os babilônios apresentassem um conhecimento prático de frações, a utilização do número zero aparece somente no século IX e os números inteiros negativos só são aceitos e compreendidos totalmente no século XVII. Com isso, percebemos as dificuldades encontradas no desenvolvimento da concepção dos números reais.

Os números irracionais aparecem em registros das civilizações egípcias e babilônicas e também eram conhecidos pelos gregos, mas foram motivo de controvérsias até o final do século XIX, quando matemáticos como R. Dedekind e G. Cantor, por exemplo, mostram como os números irracionais podem ser construídos a partir dos números racionais. Com isso, os matemáticos puderam definir de forma precisa os conceitos de limite e continuidade, contribuindo para o desenvolvimento da análise matemática.

Com a formalização da teoria dos números reais, os matemáticos e filósofos passaram a se preocupar em dar uma fundamentação axiomática consistente para a teoria de conjuntos. Com isso, uma das questões centrais passa a ser a definição de número. De fato, em 1901, G. Peano propõe a construção dos números naturais com base em um sistema de axiomas. A teoria de G. Peano e a teoria de conjuntos de Cantor incitaram matemáticos e filósofos a estudar profundamente os fundamentos da Matemática. No início do século XX, B. Russell difundiu a ideia de que a Matemática é um ramo da Lógica e sustentou tal afirmação, definindo os números naturais tomando-se por base os conceitos de lógica. Nesse mesmo período, E. Zermelo guiou um movimento para reconstruir a teoria dos conjuntos por meio da introdução de uma formulação axiomática, em substituição ao enfoque estabelecido por Cantor. John von Neumann contribuiu com esse movimento, utilizando os axiomas de Zermelo para definir os números naturais.

\section{Bibliografia}

ALKHAYYAMI, Omar. 1851. L'Algèbre D’Omar Alkhayyami. Trad. F. Woepcke. Paris: Benjamin Duprat.

ARISTOTE. 1872. Physique. Tome II. Trad. J. B. Saint-Hilaire. Paris: Ladgrange.

BALIEIRO FILHO, Inocêncio Fernandes. (2004). Arquimedes, Pappus, Descartes e Polya - Quatro Episódios da História da Heurística. Tese de Doutorado, UNESP - Universidade Estadual Paulista Júlio de Mesquita Filho (Doutorado em Educação Matemática), Rio Claro.

BALIEIRO FILHO, I. F.; SOARES, Marcelo Reicher. 2008. Uma Abordagem da Análise Matemática para Alguns Problemas Derivados das Concepções Filosóficas de Zenon, Atinfon e Brison. Revista Brasileira de História da Matemática, v. 8, p. 155-172.

BASHMAKOVA, Isabella.; SMIRNOVA, Galina. 2000. The Beginnings and Evolution of Algebra. Washington: The Mathematical Association of America.

BELNA, Jean-Pierre. 1996. La Notion de Nombre chez Dedekind, Cantor, Frege Théories, conceptions etphilosophie. Paris: Vrin. 
BESSELAAR, José VanDen. 1973. Introdução aos Estudos Históricos. São Paulo: EditoraPedagógica e Universitária Ltda.

BURTON, David.2011. The History of Mathematics: An Introduction. New York: McGrawHill.

BROWN, Stuart.; FOX, N. J. 2006. Historical Dictionary of Leibniz's Philosophy.Maryland: Scarecrow.

CANTOR, George. 1883a. Une Contribution a la Théorie des Ensembles. Acta Mathematica, v. 2, p.311-328.

CANTOR, George. 1883b. Surles Ensembles Infinis et Linéaires de Points. Acta Mathematica, v. 2, p.349-380.

CAUCHY, Augustin Louis. 1821. Coursd'Analyse de L'Ecole Royale Polytechnique. Paris: Debure.

D’ALEMBERT, Jean.;DIDEROT, Denis. 1785. EncyclopédieMéthodique. Tome 2, Paris: Debure.

DE MORGAN, Augustus. 1837. Elements of Algebra. London: Taylor and Walton.

DEDEKIND, Richard. 1901. Essay on the Theory of Numbers. Trad. W. W. Beman. Chigago: The Open Court Publishing Company.

DIOPHANTE. 1926. Les sixlivresarithmétiques et le livre des nombres polygones. Trad. P. V. Eecke. Bruges: Desclée de Brouwer.

EUCLIDES. 2009. Os Elementos. Trad. I. Bicudo. São Paulo: Fundação Editora da UNESP. EULER, Leonhard. 1774. Élémensd'Algebre. Tome Premier. Lyon: Jean-Marie Bruyset. Original: Vollständige Anleitung zur Algebra , 1770.

EWALD, Willian. 1999. From Kant to Hilbert: A Source Book in the Foundations of Mathematics. Volume I, New York: Oxford University Press.

FENNEMA, Elizabeth.;LOEF, Michael. 1992. Teacher's knowledge and its impact. Em D. A. Grouws (ed.), Handbook of research in mathematics teaching and learning. New York: Macmillan.

HEATH, Thomas. 1970. Mathematics in Aristotle. Oxford: Oxford University Press.

HEATH, Thomas. 2003. A History of Greek Mathematics. Vol 1. New York: Dover.

IVOR, Thomas. 1957. Selections Illustrating the History of Greek Mathematics. Vol. 1. Cambrigde: Harvard University Press.

KANT, Immanuel. 1905. Critique de la Raison Pure. Trad. A. Tremesaygues \& B. Pacaud. Paris: Félix Alcan, 1905.

KATZ, Victor. J. 1998. A History of Mathematics: An Introduction. New York: AddisonWesley.

KLEIN, Felix. 2004. Elementary Mathematics from an Advanced Standpoint. New York: Dover.

LEIBNIZ, Gottfried Wilhelm. 1765. Oeuevres Philosophiques. Amsterdam: Jean Schreuder. MAY, Kenneth. O. 1978. Bibliography and Research Manual of the History of Mathematics. Toronto: University of Toronto Press.

MUSA, Mohammed Ben. 1831. The Algebra of Mohammed Ben Musa. Trad. F. Rosen. London: The Oriental Translation Fund.

NEWTON, Isaac. 1720. Universal Arithmetick: or, a Treatise of Arithmetical Composition and Resolution. Trad. J. Raphson. London: J. Senex. Versão original: Aritmetica 
Universalis, sive de compositione et resolutione aritmetica liber, 1707.

PEACOCK, George. 1830. Treatise on Algebra. London: J. \& J.J. Deighton.

REALE, Giovanni.;ANTISERI, Dario. 1990. História da Filosofia, v.1. São Paulo: Paulus.

RUSSELL, Bertrand. 1919. Introduction to Mathematical Philosophy. London: George Allen \& Unwin.

STEVIN, Simon. 1585. L'Arithmetique.Leyde: ChristophlePlantin.

STRUIK, Dirk Jan. 1992. História Concisa das Matemáticas. Lisboa: Gradiva.

THOMPSON, Alba G. 1992. Teacher's beliefs and conceptions: A synthesis of the research. Em D. A. Grouws (ed.), Handbook of research in mathematics teaching and learning. New York: Macmillan.

Inocêncio Fernandes Balieiro Filho

Departamento de Matemática - UNESP - Campus

de Ilha Solteira - Brasil

E-mail:balieiro@mat.feis.unesp.br 